\title{
Forma y orientación de las manzanas urbanas en el Área Metropolitana de Mendoza en relación con el potencial energético
}

\section{Form and orientation of urban blocks in the Metropolitan Area of Mendoza related to energy potential}

\author{
Mariela E. Arboit ${ }^{1}$, Dora S. Maglione ${ }^{2}$, Manuela Fontanive ${ }^{3}$ \\ marboit@mendoza-conicet.gob.ar,dmaglione@uarg.unpa.edu.ar,fmanuela24@gmail.com \\ ${ }^{1}$ Instituto de Ciencias Humanas, Sociales y Ambientales, CCT Mendoza, CONICET, \\ Argentina \\ ${ }^{2}$ Instituto de Trabajo, Economía y Territorio Universidad Nacional de la Patagonia Austral, \\ Argentina \\ ${ }^{3}$ Laura Magistrale in Pianificazione Territoriale, Urbanistica e Paesaggistico-Ambientale, \\ Politecnico di Torino, Italia
}

Recibido: 28/05/2020. Aceptado: 14/12/2020

\section{RESUMEN}

El consumo de la energía en la edificación se ve incrementado por el aumento de la población urbana, la fuerte expansión de las ciudades y las actividades antropogénicas, especialmente en los países en desarrollo, siendo principalmente dependiente de las condiciones del clima urbano y de la morfología urbano-edilicia. El estudio tiene como objetivo profundizar el conocimiento de las variables forma y orientación de las manzanas urbanas ya que las mismas intervienen en el potencial solar de entornos urbanos típicos del área metropolitana de Mendoza (AMM), condicionando la posibilidad de utilizar estrategias de diseño bioclimático y limitando la posibilidad de acceso a los recursos que ofrece el clima en distintas épocas del año.

Metodológicamente, se calculó la orientación del eje principal para la totalidad de las manzanas urbanas del AMM, en sistemas de información geográfica (SIG), y de su forma como proporción de lados de las unidades predominantemente rectangulares, lo que permitieron obtener resultados y desarrollar modelos estadísticos. Los resultados presentan limitaciones propias relacionadas con la orientación principalmente en áreas de crecimiento y densificación urbana que están llevando a una situación crítica tanto en lo ambiental como en lo energético. La investigación permitirá a futuro identificar e implementar propuestas de crecimiento y renovación urbana indispensables para una aproximación a la consecución de la sostenibilidad ambiental y energética del tejido urbano, definiendo lineamientos y estrategias.

Palabras clave: morfología urbano-edilicia; planificación urbana; energías renovables.

\begin{abstract}
The energy consumption in the building is increased by the increase of the urban population, the strong expansion of cities and anthropogenic activities, especially in developing countries, being mainly dependent on the conditions of the urban climate and morphology urbanedilicia. The study aims to deepen the knowledge of the variables urban form and orientation since it intervenes in the solar potential of urban environments typical of the metropolitan area of Mendoza (AMM), conditioning the possibility of using bioclimatic design strategies and
\end{abstract}


limiting the possibility of access to the resources offered by the climate at different times of the year.

Methodologically, the orientation of the main axis for all the buildings constructed for the urban blocks of the AMM and its shape (as a proportion of sides of the predominantly rectangularunits) was calculated, using the geographic information systems (GIS). These informations allowed obtaining results and developing statistical models. The results present own limitations related to the orientation mainly in the areas of urban growth and densification that are leading to a critical situation both environmentally and energyly. The research will allow the future to identify and implement proposals for urban growth and renewal that are essential for an approach to the achievement of the environmental and energy sustainability of the urban fabric, defining guidelines and strategies.

Keywords: urban morphology-edilicia; urban planning; renewable energy.

\section{INTRODUCCIÓN}

Entre los problemas más críticos que se debe enfrentar en la búsqueda del desarrollo sostenible se encuentran los interrelacionados correspondientes al hábitat y la energía. El primero constituye el continente espacial de toda actividad humana y produce el impacto más significativo y durable de dicha actividad sobre el medio natural. La energía por otra parte, es un insumo indispensable para el funcionamiento y la supervivencia de la sociedad urbana y es imperativo confrontar, durante el presente siglo, una difícil transición desde el consumo masivo de combustibles fósiles agotables y contaminantes a fuentes alternativas renovables y limpias (Owens, 1986; Givoni, 1998, Breheny, 1996; Droege, 2007; Jones, Williams \& Lannon, 2000; Ratti, Raydan \& Steemers, 2003; Steemers, 2003).

Dentro del sinnúmero de problemas ambientales que afectan a las estructuras urbanas regionales, el presente estudio se concentrará en dos de ellos cuyo carácter es indiscutiblemente crítico. En primer lugar, en lo relativo a lo energético, el único recurso disponible en el medio urbano para su aprovechamiento irrestricto en el Área Metropolitana de Mendoza (AMM) es el recurso solar. Las posibilidades de uso son: la calefacción solar de espacios, pasiva y activa, el calentamiento de agua para uso doméstico y la generación fotovoltaica (Basso, Fernández, Mesa, Córica \& de Rosa, 2003). En trabajos previos se ha estudiado exhaustivamente las implicancias de la morfología urbana para posibilitar el uso del recurso solar en entornos urbanos de baja y alta densidad (Arboit, Diblasi, Fernández Llano \& Rosa, 2008; Arboit, Mesa, Diblasi, Fernandez Llano \& de Rosa, 2010; Arboit \& de Rosa, 2013). Sin embargo, es aún necesario abordar aspectos específicos para poder definir los lineamientos técnicos transferibles a municipios y a la provincia, para actualizar Leyes de Loteo, Códigos Urbanos y de Edificación. En segundo término, relacionado a lo ambiental, se considera necesario comenzar a legislar y promover el reciclaje energético de construcciones existentes, mediante el mismo podría lograrse una recuperación parcial del yacimiento energético subyacente, una recuperación de materiales y componentes posible de reutilizar o reciclar y finalmente, una menor emisión de residuos sólidos, de difícil absorción por el medio ambiente.

El conocimiento de datos y características del ambiente urbano construido (de la morfología urbana), está en estrecha relación con las condiciones energéticas y el confort urbano; otro aspecto importante es la infraestructura, para poder relacionar lo físico y lo bioclimático en la planificación de una ciudad energéticamente más eficiente. En el Área Metropolitana de Mendoza (AMM) el tejido urbano (estudiado previamente en formato CAD; Arboit, de Rosa, 2013b), está integrado por la geometría de las manzanas, la morfología edilicia y la 
infraestructura verde. En la descripción de la morfología del espacio urbano se consideran una serie de factores relacionados a la calidad del ambiente, se evalúa la influencia de la morfología urbana sobre la disponibilidad de radiación solar, que permita entender las condiciones energéticas y el comportamiento bioclimático.

Recientemente para el AMM se ha modelado la distribución espacial del consumo de energía (gas por red) residencial a escala urbana (Mutani, Fontanive \& Arboit 2018), quedando pendiente el objetivo de profundizar el conocimiento de las posibilidades de maximizar la eficiencia energética de los edificios urbanos, considerando las variables urbanas principales, entre ellas la forma y orientación de las manzanas urbanas, a fin de completar los resultados de la investigación, extraer conclusiones y elaborar estrategias que permitan a futuro generar propuestas normativas transferibles que tiendan a mejorar las posibilidades de mayor eficiencia energética en los edificios urbanos.

\section{CASO DE ESTUDIO}

Se ha definido como universo de análisis el Área Metropolitana de Mendoza (AMM), que se ubica a $32.85^{\circ}$ de latitud Sur, $68.85^{\circ}$ longitud Oeste y 870 m.s.n.m., desarrollada sobre un oasis de cultivo con riego artificial, lindando su borde oeste con el piedemonte andino.

El AMM es el conglomerado urbano más importante de la región, conformado por seis departamentos administrativos: Capital, Godoy Cruz, Guaymallén, Las Heras, Luján de Cuyo y Maipú, dentro del denominado Oasis Norte (Figuras 1 y 2). Mendoza urbana está integrada por la geometría de las manzanas, la morfología de los volúmenes construidos, las áreas verdes, acequias y arboleda. Presenta inviernos templado-fríos, cuenta con un generoso recurso solar $\left(4.58 \mathrm{kWh} / \mathrm{m}^{2}\right.$ día a $5.55 \mathrm{kWh} / \mathrm{m}^{2}$ ) y con predominio de cielos claros (Servicio Meteorológico Nacional, 2019).

Para la definición de requerimientos de climatización o estrategias de diseño, se consideran los grados día anuales de calefacción y enfriamiento, como la suma de las diferencias entre la temperatura base (TB) de confort interior y la temperatura media exterior del día, con valores para el $\mathrm{AMM}$ de $1384^{\circ} \mathrm{C}$ día anuales de calefacción $\left(\mathrm{TB} 18^{\circ}\right.$ ) y $163^{\circ} \mathrm{C}$ día anuales de enfriamiento $\left(\mathrm{TB} 23^{\circ}\right)$. 


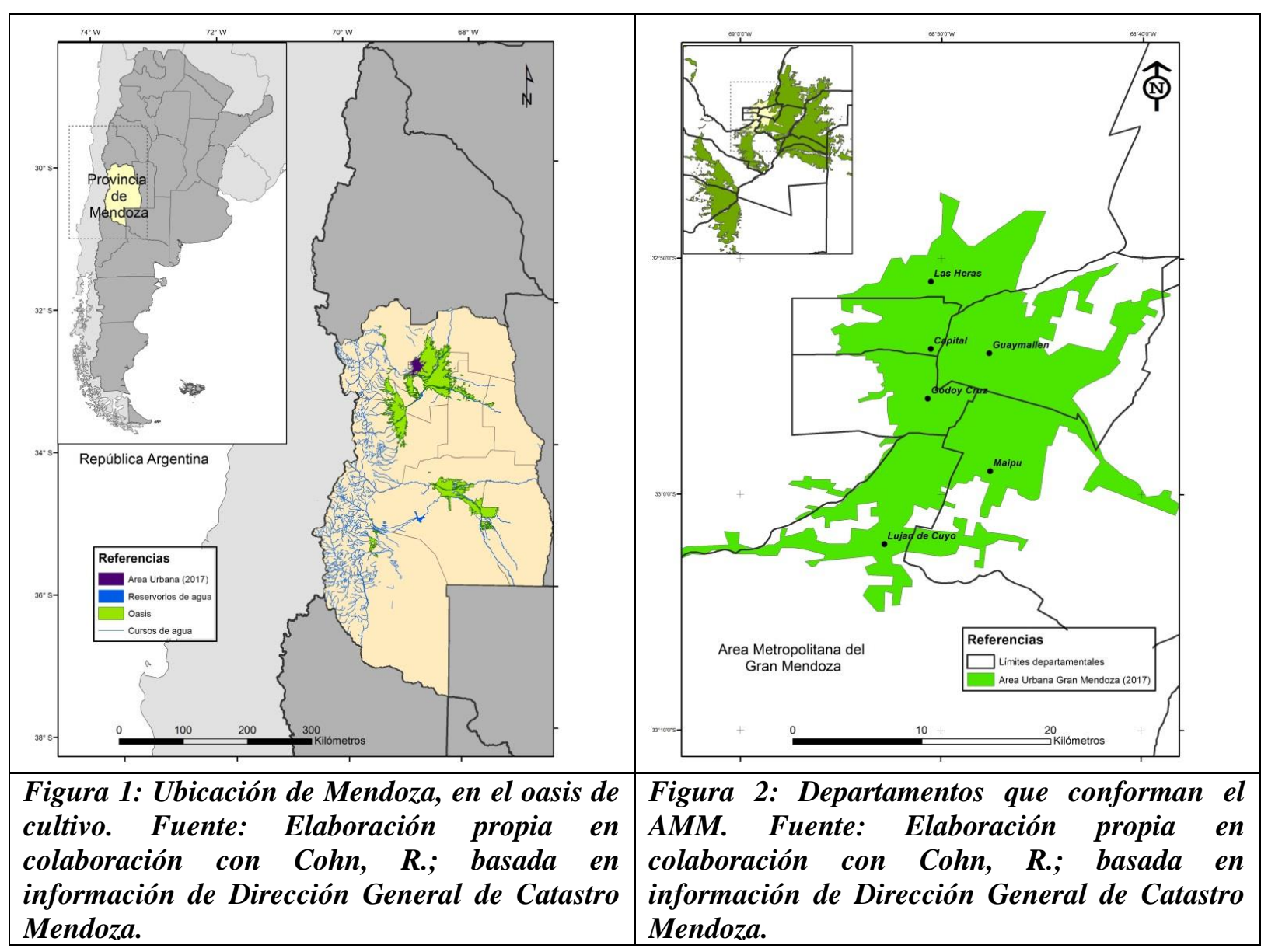

Se han considerado el estudio de las manzanas de forma ortogonal ya que, desde el punto de vista cuantitativo, son las más dominantes en los entornos urbanos construidos del AMM, no sólo por su extensión sino también porque soportan los tejidos de mayor densidad edilicia (Figura 3).

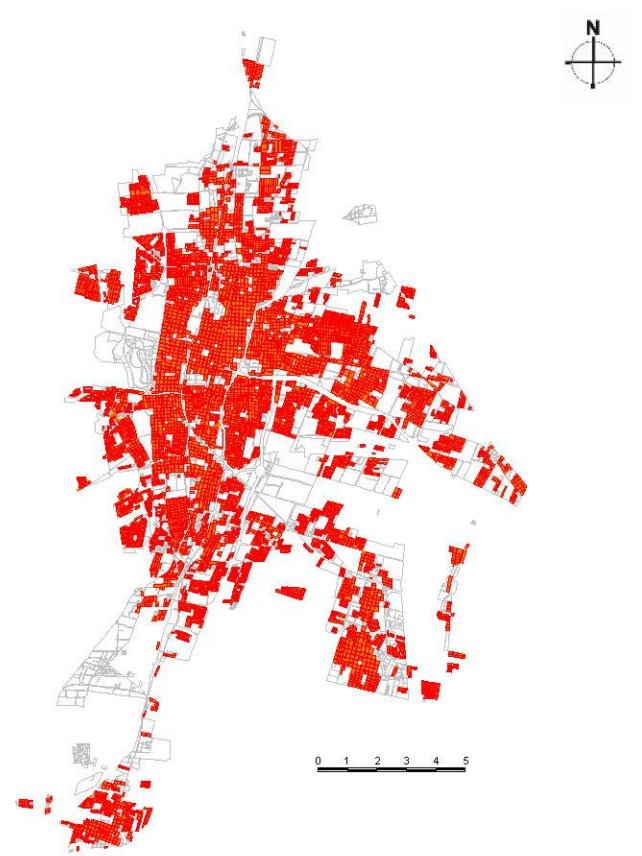

Figura 3: Manzanas urbanas ortogonales. Fuente: Elaboración propia 


\section{METODOLOGÍA}

La investigación se desarrolló en base a la recopilación de la información cartográfica disponible en entorno GIS y del análisis de datos catastrales urbano-edilicios (Dirección Provincial de Catastro, 2010, actualizada al 2012 para Capital y Guaymallén). Se identificaron y cuantificaron las características principales del trazado urbano:

1. Forma de las manzanas: Está referida a la proporción de los lados de manzanas aproximadamente rectangulares. Según dichas proporciones se determinan 5 (cinco) tipos y se los identifica mediante sus proporciones de largo (L) y ancho (A): Categoría 1: < 1.25 (cuadradas); Categoría 2: 1.25 a 2.25; Categoría 3: 2.25 a 3.25; Categoría 4: 3.25 a 4.25; Categoría 5: > 4.25 (Figura 4). Las manzanas con formas más irregulares que corresponden a loteos periféricos, no edificados, mayores a un área de <30000 o con una relación $\mathrm{L} / \mathrm{A}>=10$ fueron considerados como errores de la base de datos y se descartan a fin de tener datos homogéneos, los mismos han sido clasificados como Categoría 0 (manzanas no consideradas para el análisis).

2. Orientación de las manzanas: Se refiere al ángulo formado por el lado más largo de la manzana y la directriz N-S. Se determinaron 12 tipos en tramos de $15^{\circ}$ cada uno, resultando $90^{\circ}$ para una manzana cuyo lado más largo está en la dirección E-O. Categoría 1: de $75^{\circ}$ a $105^{\circ}$ medido desde la orientación Norte (orientación óptima); Categoría 2: de $105^{\circ}$ a $120^{\circ}$ y de $60^{\circ}$ a $75^{\circ}$; Categoría 3: de $120^{\circ}$ a $135^{\circ}$ y de $45^{\circ}$ a $60^{\circ}$; Categoría 4: de $135^{\circ}$ a $150^{\circ}$ y de $30^{\circ}$ a $45^{\circ}$; Categoría 5: de $150^{\circ}$ a $165^{\circ}$ y de $15^{\circ}$ a $30^{\circ}$; Categoría 6: de $165^{\circ}$ a $180^{\circ}$ y de $0^{\circ}$ a $15^{\circ}$ (orientación desfavorable). La Categoría 0: son las manzanas no consideradas para el análisis (Figura 5).

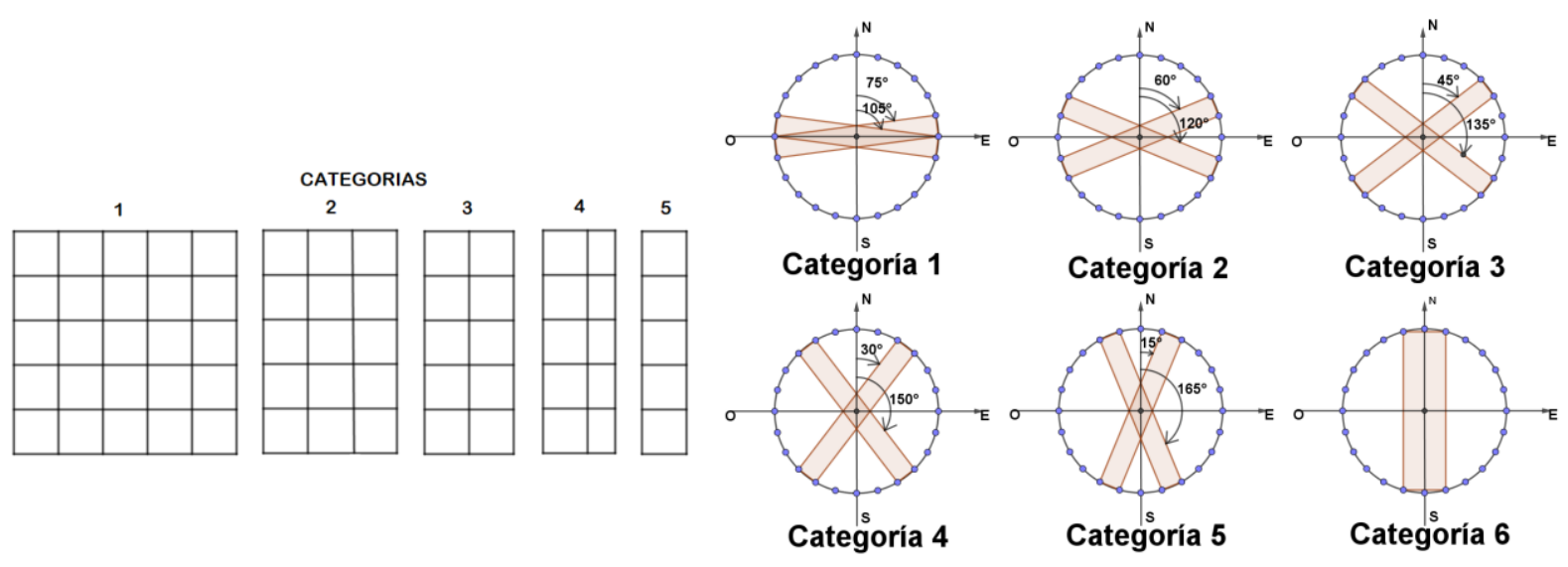

Figura 4: Forma de las manzanas.

Fuente: Elaboración propia.

Figura 5: Orientación de las manzanas. Fuente:

Elaboración propia.

Para el análisis de orientación de las manzanas de forma cuadrada Categoría 1: $<1.25$ se han considerado solo tres categorías de orientación agrupando: en Categoría 1 (orientación óptima) de $75^{\circ}$ a $105^{\circ}$, de $165^{\circ}$ a $180^{\circ}$ y de $15^{\circ}$ a $0^{\circ}$; en Categoría 2: de $105^{\circ}$ a $120^{\circ}$, de $60^{\circ}$ a $75^{\circ}$, de $150^{\circ}$ a $165^{\circ}$ y de $15^{\circ}$ a $30^{\circ}$ y en Categoría 3: de $120^{\circ}$ a $135^{\circ}$, de $45^{\circ}$ a $60^{\circ}$, de $135^{\circ}$ a $150^{\circ}$ y de $30^{\circ}$ a $45^{\circ}$. Esta agrupación de categorías de las manzanas cuya proporción entre sus lados es $<1.25$ se ha realizado ya que presentan valores constantes de áreas colectoras independientes de la orientación, por ejemplo, las áreas colectoras de las manzanas orientadas de $75^{\circ}$ a $105^{\circ}$ comparada con las orientadas de $165^{\circ}$ a $180^{\circ}$ y de $0^{\circ}$ a $15^{\circ}$ son equivalentes al rotar $90^{\circ}$ dichas manzanas (igualando la forma de manzana en la Categoría 1 y 6). La misma situación se repite en la forma de manzanas Categoría 2 con 5 y Categoría 3 con 4. 
Los datos del trazado urbano se procesaron en programación GIS, considerando el número de manzanas. El cálculo de largo, ancho de manzanas y orientación del eje principal permitió obtener los valores de forma y orientación para la totalidad de las manzanas urbanas analizadas (10390).

A partir de los datos medios por manzana y para las categorías definidas se calcularon distintas medidas resúmenes y las correlaciones entre las variables, se compararon los valores medios de cada departamento usando análisis de varianza, se construyeron gráficos de densidades a partir de las categorías definidas sobre el forma y orientación de manzanas y se utilizó el test chi-cuadrado para independencia de variables categóricas. El análisis estadístico se realizó usando los software Infostat (Di Rienzo et al, versión 2018) y R (R Core Team, 2018).

\section{RESULTADOS}

\subsection{Forma de las manzanas en el AMM}

En las Figuras 6 y 7 se muestran los resultados de valores de la forma de la manzana urbana, para las 10390 manzanas urbanas analizadas, y en la Tabla 1 algunas medidas resúmenes de los valores de forma de manzana. La mediana de forma de manzana es $2.11 \mathrm{y}$ el $50 \%$ de los datos centrales están entre 1.49 y 3.05 .
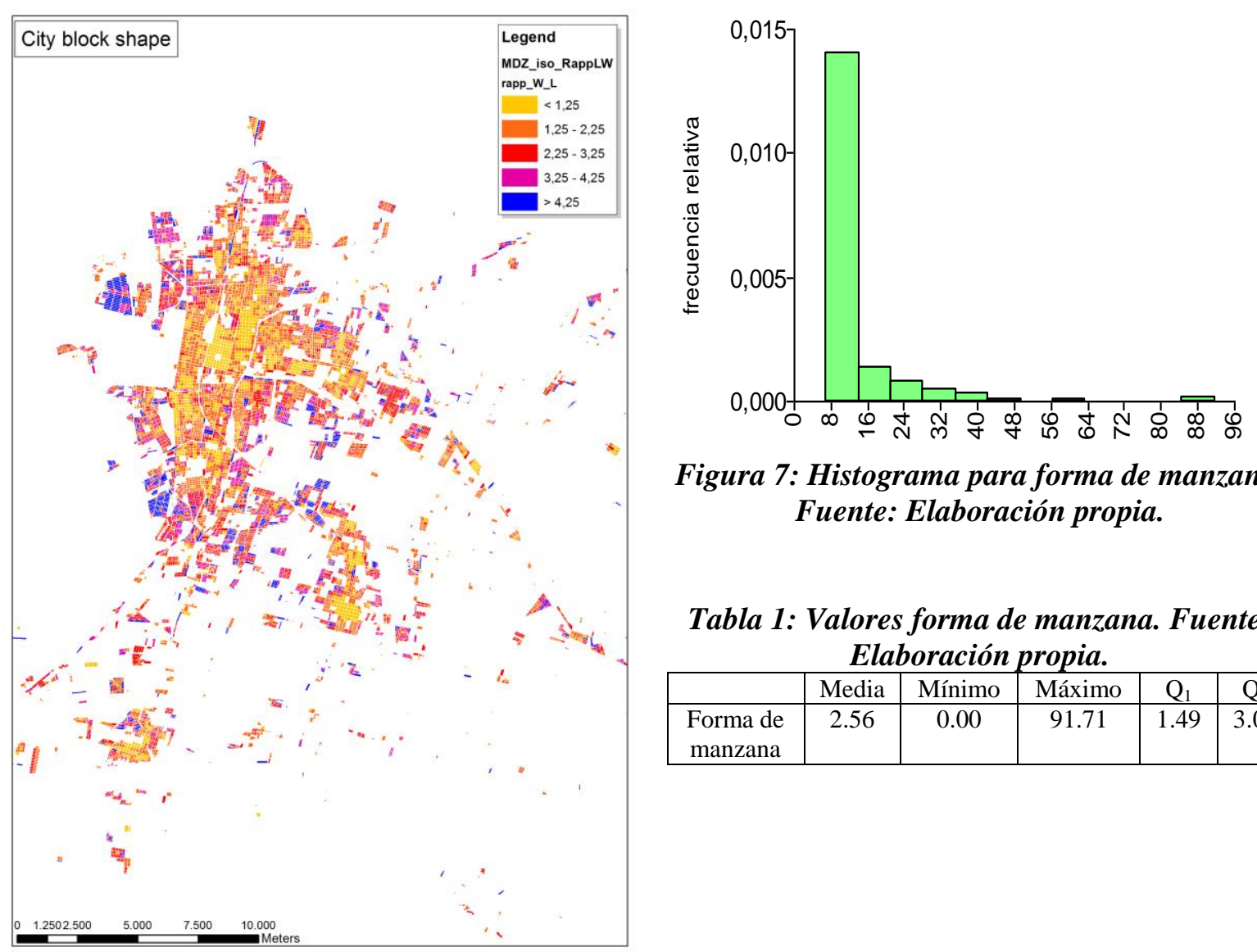

Figura 7: Histograma para forma de manzana. Fuente: Elaboración propia.

Tabla 1: Valores forma de manzana. Fuente: Elaboración propia.

\begin{tabular}{|c|c|c|c|c|c|}
\hline & Media & Mínimo & Máximo & $\mathrm{Q}_{1}$ & $\mathrm{Q}_{3}$ \\
\hline $\begin{array}{c}\text { Forma de } \\
\text { manzana }\end{array}$ & 2.56 & 0.00 & 91.71 & 1.49 & 3.05 \\
\hline
\end{tabular}

Figura 6: Valores de forma de la manzana urbana. Fuente: Elaboración propia. 


\subsubsection{Análisis categorizado de forma de manzana}

En la Figura 8 y 9 se presentan gráficos de puntos y de barras para la categorización de las manzanas urbanas en las tipologías definidas anteriormente.

De las 10390 manzanas urbanas con datos catastrales del AMM la categoría con mayor representatividad corresponde a la Categoría 2 con un $40.89 \%$, seguida por la Categoría 3 (23.90\%), la Categoría 1 (13.83\%), la Categoría $4(11.70 \%)$ y la Categoría $5(9.60 \%)$ que es la de menor representatividad. Para la Categoría 2 el valor medio es 1.71 y el $50 \%$ de las manzanas presentan valores entre 1-47 y 1.96. (Tabla 2).
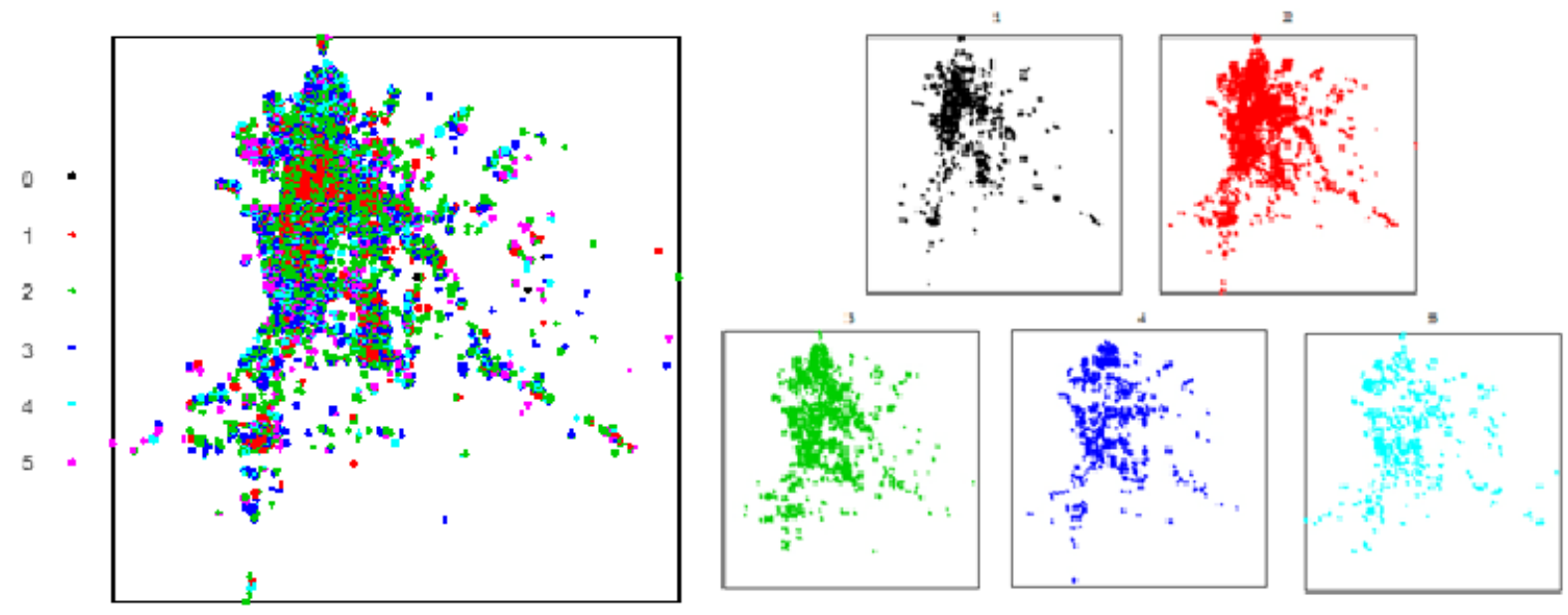

Figura 8: Gráfico de puntos para las categorías definidas para forma de manzana. Fuente: Elaboración propia.

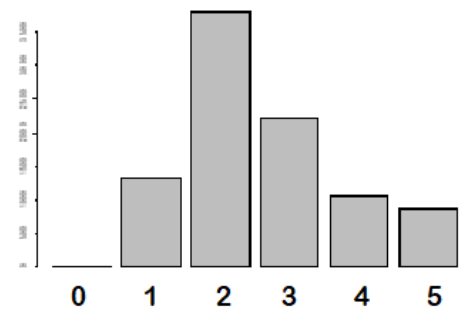

Figura 9: Categorías definidas de forma de manzana. Fuente: Elaboración propia.

Tabla 2: Valores categorizados para forma de manzana. Fuente: Elaboración propia.

\begin{tabular}{|l|c|l|l|l|l|l|}
\hline \multirow{2}{*}{ Categoría } & \multicolumn{7}{|c|}{ Valores categorizados } & & \\
\cline { 2 - 7 } & $\mathrm{N}^{\circ}$ & Media & Mínimo & Máximo & $\mathrm{Q}_{1}$ & $\mathrm{Q}_{3}$ \\
\hline 1 & 1437 & 1.12 & 0 & 1.25 & 1.05 & 1.19 \\
\hline 2 & 4248 & 1.71 & 1.25 & 2.25 & 1.47 & 1.96 \\
\hline 3 & 2483 & 2.70 & 2.25 & 3.25 & 2.45 & 2.96 \\
\hline 4 & 1216 & 3.68 & 3.25 & 4.25 & 3.44 & 3.91 \\
\hline 5 & 997 & 6.57 & 4.25 & 91.71 & 4.58 & 6.36 \\
\hline
\end{tabular}

Los histogramas de cada categoría infieren que los valores de las variables se distribuyen en forma aproximadamente uniformemente dentro de las categorías que existen (Figura 10). En la Figura 11 se observa un gráfico continuo, en donde visualmente se detectan las áreas con concentración de manzanas categorizadas. 

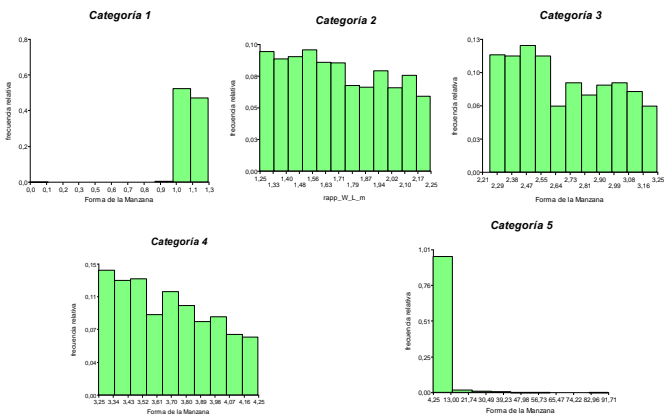

Figura 10: Histograma para cada categoría de forma de manzana. Fuente: Elaboración propia.

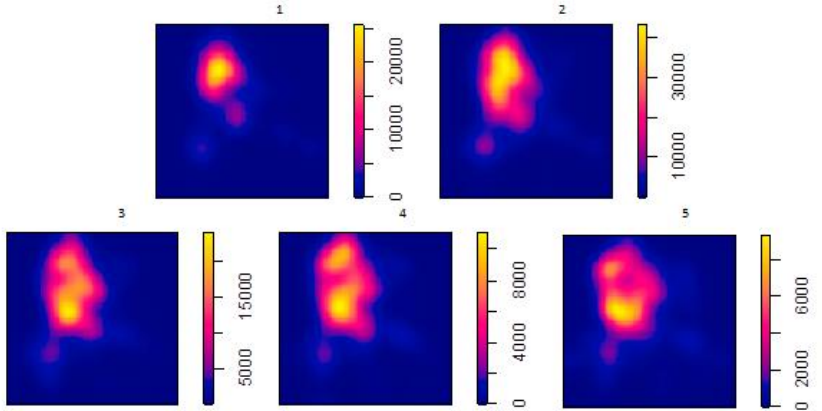

Figura 11: Análisis de densidad, número de manzanas categorizadas por unidad de área. Fuente: Elaboración propia.

\subsubsection{Análisis por departamento de forma de manzanas}

Los resultados para todos los departamentos indican valores medios de forma de manzana predominantes para la Categoría 2. Se ofrece un resumen de los valores categorizados por departamento (Tabla 3). Usando un análisis de la varianza no paramétrico (Kruskal-Wallis), existe diferencia entre valores medios de los departamentos ( $p$-valor $<<0.0001)$. De los seis departamentos del AMM, el valor medio más alto (2.91) se ubicó en Maipú seguido por Godoy Cruz (2.69). En valores absolutos los departamentos con valores de forma de manzanas más bajos son Capital y Guaymallén (2.31 y 2.33 respectivamente). Al comparar las medias entre departamentos se observa que los valores medios de Capital y Guaymallén no son significativamente distintos (Tabla 4).

Tabla 3: Resumen valores de forma de manzanas por departamento. Fuente: Elaboración propia.

\begin{tabular}{|l|c|c|c|c|c|c|}
\hline Departamento & $\mathrm{N}^{\circ}$ & Media & Mín & Máx & $\mathrm{Q}_{1}$ & $\mathrm{Q}_{3}$ \\
\hline Capital & 1221 & 2.31 & 1.00 & 22.93 & 1.33 & 2.75 \\
\hline Godoy Cruz & 2092 & 2.69 & 0.00 & 91.71 & 1.59 & 3.23 \\
\hline Guaymallén & 2557 & 2.33 & 1.00 & 40.14 & 1.35 & 2.83 \\
\hline Las Heras & 1676 & 2.56 & 1.00 & 31.12 & 1.55 & 3.17 \\
\hline Luján de Cuyo & 1098 & 2.58 & 1.00 & 26.34 & 1.53 & 3.02 \\
\hline Maipú & 1746 & 2.91 & 0.00 & 90.21 & 1.62 & 3.18 \\
\hline
\end{tabular}

\section{Tabla 4: Forma de manzanas. Fuente: Elaboración propia.}

* Medias con una letra común no son significativamente diferentes $(p>0,01)$

\begin{tabular}{|l|c|c|c|}
\hline Departamento & \multicolumn{3}{|c|}{$*$} \\
\hline Capital & A & & \\
\hline Guaymallén & A & & \\
\hline Luján de Cuyo & & B & \\
\hline Las Heras & & B & C \\
\hline Godoy Cruz & & & C \\
\hline Maipú & & & C \\
\hline
\end{tabular}

El p-valor del test chi-cuadrado, indica que las variables de departamento y forma de las manzanas están asociadas, es decir que la distribución de la forma de la manzana depende del departamento que se esté considerando (se excluye la Categoría 0), (Tabla 5).

Tabla 5: Frecuencias absolutas. Fuente: Elaboración propia.

\begin{tabular}{|l|c|c|c|c|c|c|c|}
\hline Departamento & 0 & 1 & 2 & 3 & 4 & 5 & Total \\
\hline Capital & 0 & 252 & 532 & 229 & 104 & 104 & 1221 \\
\hline Godoy Cruz & 5 & 202 & 812 & 562 & 262 & 249 & 2092 \\
\hline Guaymallén & 0 & 492 & 1096 & 523 & 247 & 199 & 2557 \\
\hline Las Heras & 0 & 176 & 699 & 402 & 262 & 137 & 1676 \\
\hline Luján de Cuyo & 0 & 144 & 453 & 272 & 118 & 111 & 1098 \\
\hline Maipú & 4 & 171 & 656 & 495 & 223 & 197 & 1746 \\
\hline Total & 9 & 1437 & 4248 & 2483 & 1216 & 997 & 10390 \\
\hline
\end{tabular}


Capital posee el $20.6 \%$ de las manzanas con forma cuadrada (menores valores), seguida por Guaymallén (19.2\%). El porcentaje de manzanas con tipología más alargada $>4.25$ se ubican en Godoy Cruz (11.9\%), seguido por Maipú (11.3\%) y Luján de Cuyo (10.1\%), con mayores valores de forma de manzana. La Categoría 2 posee la mayor representatividad en todos los departamentos entre el $43.6 \%$ en Capital y un $37.7 \%$ en Maipú de las manzanas urbanas se ubican en esta categoría. Las manzanas no consideradas para el estudio fueron 9 (5 ubicadas en Godoy Cruz y 4 en Maipú).

\subsection{Orientación de las manzanas en el AMM}

Los resultados de valores de la orientación de la manzana urbana para las 10390 manzanas urbanas se muestran en las Figuras 12 y 13 y en la Tabla 6. La mediana de orientación de Manzana es 87.00 y el $50 \%$ de los datos centrales están entre 16.80 y 103.40

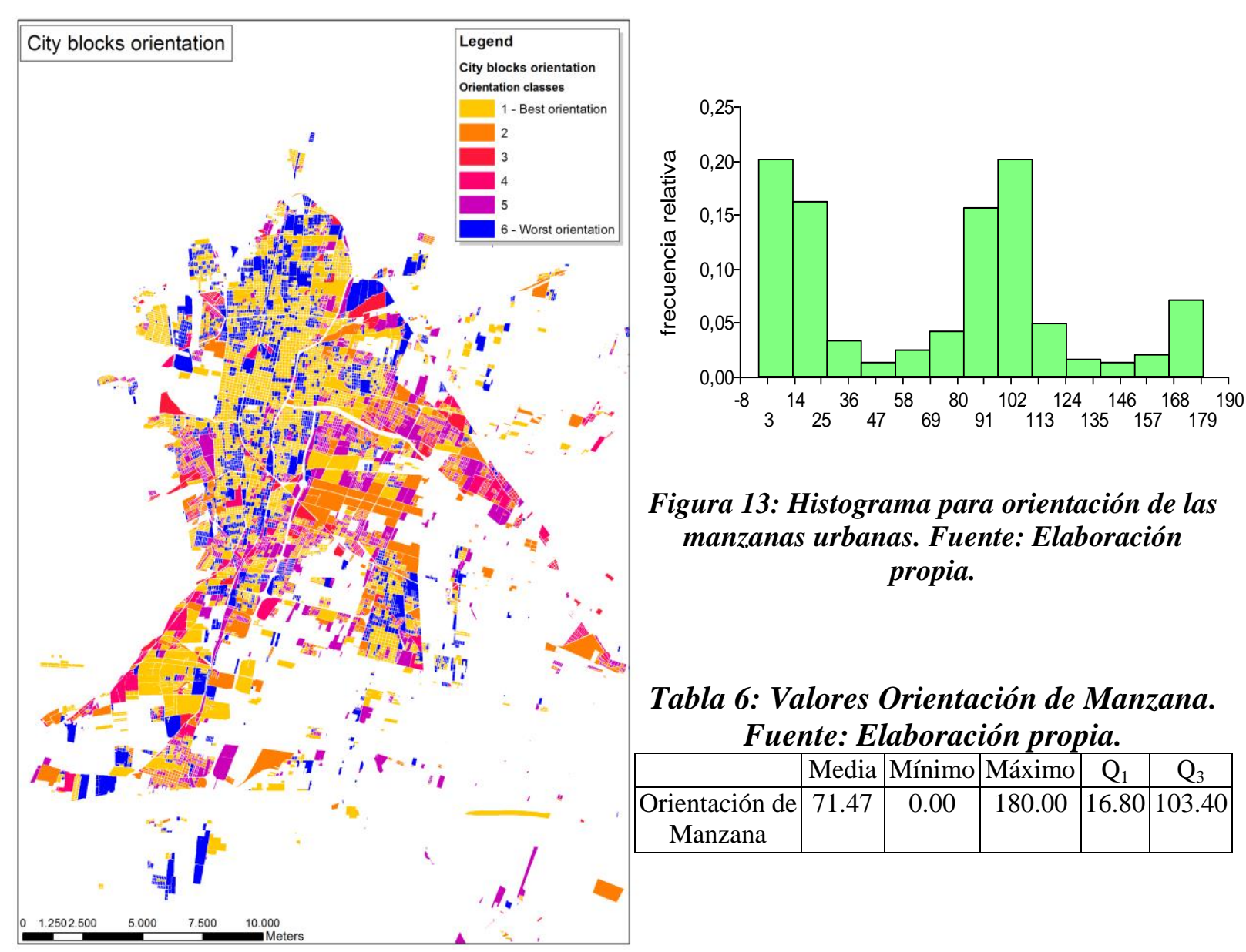

Figura 12: Valores de orientación de las manzanas urbanas. Fuente: Elaboración propia.

\subsubsection{Análisis Categorizado de orientación de manzana}

En la Figura 14 se presentan gráficos de puntos para la categorización de las manzanas urbanas en función de los valores de orientación de la manzana urbana considerando 6 tipos en tramos de $15^{\circ}$ cada uno, más la Categoría 0. Y en la Figura 15, un gráfico de barras para las categorías antes mencionadas. 
De las 10390 manzanas urbanas con datos catastrales del AMM, no se consideraron 9 manzanas $(0.09 \%)$. La categoría con mayor representatividad de manzanas urbanas es la Categoría 1 con un $39.06 \%$ de manzanas (valor medio $87.63^{\circ}$ ) el $50 \%$ de las manzanas que están en esa categoría presentan valores entre $86.80^{\circ}$ y $101.60^{\circ}$. Seguida por la Categoría 6 con un $24.53 \%$ y la Categoría 5 con $15.67 \%$ de las manzanas urbanas. Las Categoría 3 y 4 representan el $7.65 \%$ (Tabla 7).
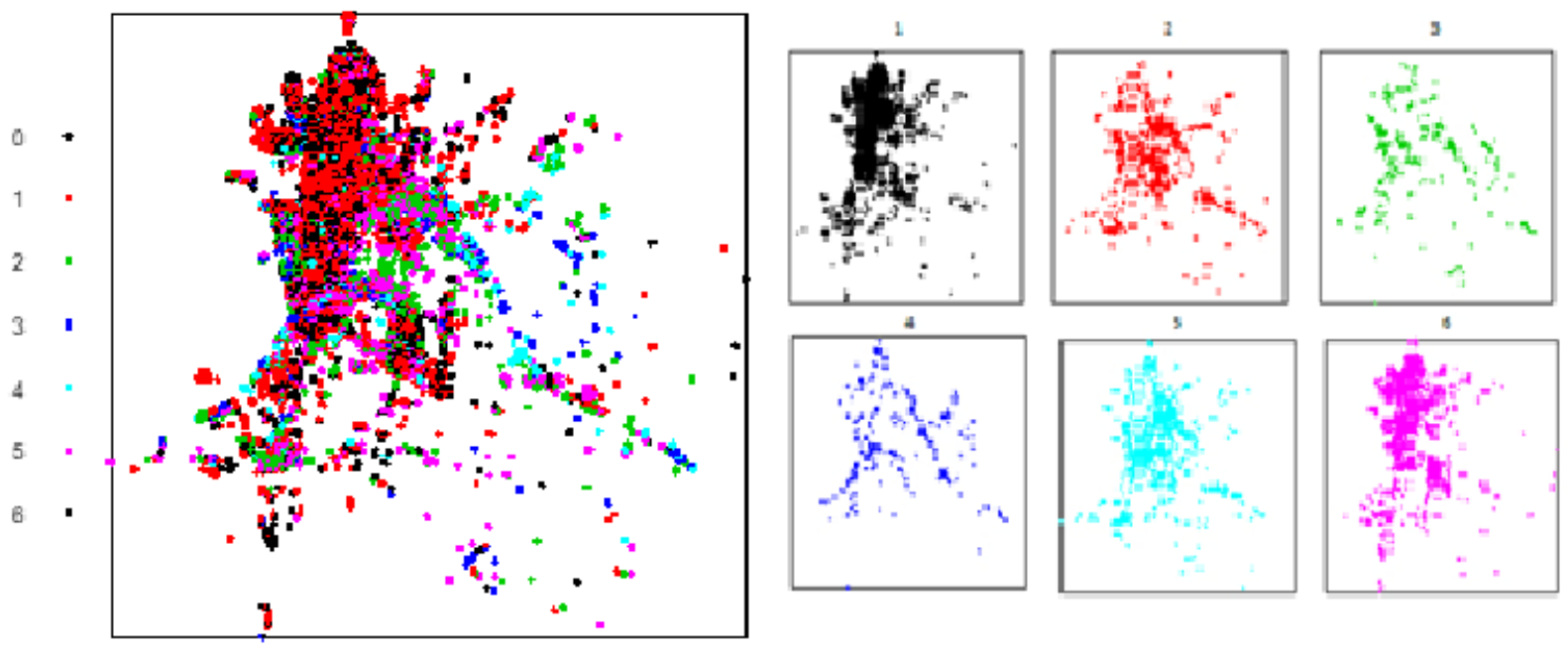

Figura 14: Gráfico de puntos para las categorías definidas para orientación de manzana. Fuente: Elaboración propia.

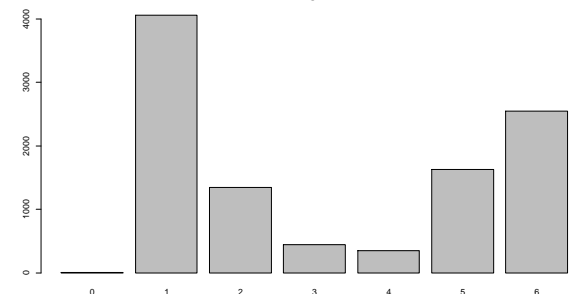

Figura 15: Categorías definidas de orientación de manzana. Fuente: Elaboración propia.
Tabla 7: Valores categorizados para orientación de manzana. Fuente: Elaboración propia.

\begin{tabular}{|l|l|l|l|l|l|l|}
\hline \multirow{2}{*}{ Cat. } & \multicolumn{5}{|c|}{ Valores categorizados } & \multicolumn{2}{c|}{} \\
\cline { 2 - 5 } & $\mathrm{N}^{\circ}$ & Med & Mín & Máx & $\mathrm{Q}_{1}$ & $\mathrm{Q}_{3}$ \\
\hline 1 & 4058 & 87.63 & 0.10 & 179.90 & 86.80 & 101.60 \\
\hline 2 & 1351 & 92.05 & 15.00 & 163.30 & 68.40 & 112.40 \\
\hline 3 & 441 & 97.65 & 30.10 & 149.20 & 54.00 & 129.80 \\
\hline 4 & 354 & 74.00 & 30.00 & 148.70 & 35.30 & 140.30 \\
\hline 5 & 1628 & 37.16 & 15.00 & 164.90 & 18.00 & 25.20 \\
\hline 6 & 2549 & 52.10 & 0.00 & 180.00 & 6.90 & 165.80 \\
\hline
\end{tabular}

En las Categorías 4, 5 y 6 se observa mayor cantidad de manzanas con orientación noreste (valores menores) que con orientación noroeste (Figura 16).

En la Figura 17 se observa un gráfico continuo, en donde visualmente se detectan las áreas con concentración de manzanas categorizadas. En las Categorías 3 y 4 hay una distribución espacial de datos, sin tanta concentración en algunas áreas. 

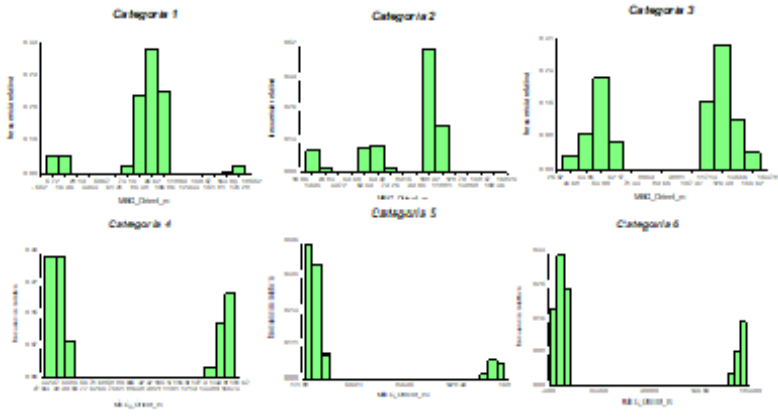

Figura 16: Histograma para cada categoría de orientación de manzana. Fuente: Elaboración propia.
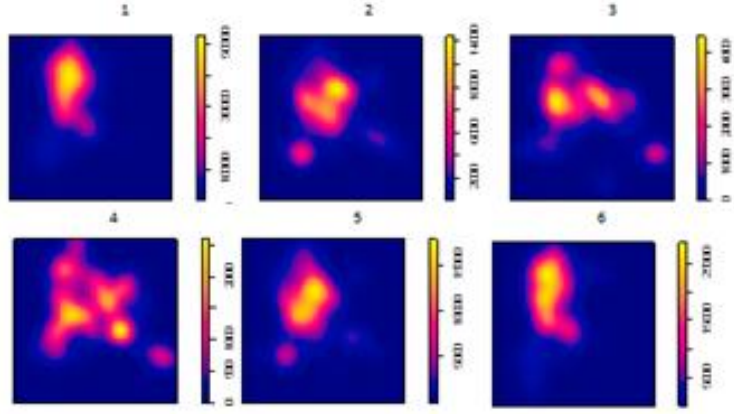

Figura 17: Análisis de densidad, número de manzanas categorizadas por área. Fuente: Elaboración propia.

\subsubsection{Análisis por departamento de la orientación de manzanas}

Los resultados para todos los departamentos indican valores medios de orientación de manzana predominantes para la Categoría 1, seguida por la Categoría 6. Se ofrece un resumen de los valores medios categorizados por departamento (Tabla 8). Usando un análisis de la varianza no paramétrico (Kruskal-Wallis) y excluyendo la Categoría 0, existe diferencia entre valores medios de orientación de manzana entre los departamentos (p-valor< <0.0001). De los seis departamentos del AMM, el valor medio más alto $\left(80.63^{\circ}\right)$ por departamento se ubica en Las Heras. En valores absolutos las manzanas urbanas con valores de orientación más bajos se ubican en Luján de Cuyo $\left(62.52^{\circ}\right)$. Comparando los valores medios por departamento se observa que los valores medios de Luján y Las Heras son significativamente distintos al resto de los departamentos. Las orientaciones de manzanas de Maipú y Capital no son significativamente diferentes entre ellas al igual que Guaymallén y Godoy Cruz (Tabla 9).

Tabla 8: Resumen valores de orientación de manzanas por departamento. Fuente: Elaboración propia.

\begin{tabular}{|l|c|l|c|c|c|c|}
\hline Departamento & $\mathrm{N}^{\circ}$ & Media & Mín & Máx & $\mathrm{Q}_{1}$ & $\mathrm{Q}_{3}$ \\
\hline Capital & 1221 & 67.87 & 0.10 & 180.00 & 12.80 & 102.50 \\
\hline Godoy Cruz & 2092 & 73.77 & 0.00 & 179.90 & 21.60 & 106.00 \\
\hline Guaymallén & 2557 & 71.92 & 0.20 & 179.90 & 17.30 & 102.70 \\
\hline Las Heras & 1676 & 80.63 & 0.00 & 179.90 & 16.00 & 102.30 \\
\hline $\begin{array}{l}\text { Luján de } \\
\text { Cuyo }\end{array}$ & 1098 & 62.52 & 0.10 & 179.40 & 15.30 & 103.20 \\
\hline Maipú & 1746 & 67.38 & 0.00 & 179.90 & 17.90 & 104.20 \\
\hline
\end{tabular}

Tabla 9: Orientación de manzanas. Fuente: Elaboración propia.

*Medias con una letra común no son significativamente diferentes $(p>0,01)$

\begin{tabular}{|l|l|l|l|l|}
\hline Departamento & \multicolumn{2}{|l|}{$*$} & \multicolumn{3}{l|}{} \\
\hline Luján & A & & & \\
\hline Maipú & & B & & \\
\hline Capital & & B & & \\
\hline Guaymallén & & & C & \\
\hline Godoy Cruz & & & C & \\
\hline Las Heras & & & & D \\
\hline
\end{tabular}

El p-valor para el test chi-cuadrado es $<0.0001$, lo que indica que las variables categorizadas de orientación de la manzana y los departamentos están asociadas, es decir que la distribución de orientaciones de manzanas depende del departamento que se esté considerando (se excluyó la Categoría 0 del análisis). 
Tabla 10: Frecuencias absolutas. Fuente: Elaboración

propia.

\begin{tabular}{|l|c|c|c|c|c|c|c|c|}
\hline Departamento & 0 & 1 & 2 & 3 & 4 & 5 & 6 & Total \\
\hline Capital & 0 & 629 & 97 & 12 & 20 & 117 & 346 & 1221 \\
\hline Godoy Cruz & 5 & 607 & 382 & 154 & 70 & 408 & 466 & 2092 \\
\hline Guaymallén & 0 & 1085 & 319 & 102 & 77 & 426 & 548 & 2557 \\
\hline Las Heras & 0 & 832 & 70 & 42 & 34 & 135 & 563 & 1676 \\
\hline Luján de Cuyo & 0 & 344 & 211 & 41 & 39 & 192 & 271 & 1098 \\
\hline Maipú & 4 & 561 & 272 & 90 & 114 & 350 & 355 & 1746 \\
\hline Total & 9 & 4058 & 1351 & 441 & 354 & 1628 & 2549 & 10390 \\
\hline
\end{tabular}

Capital y Las Heras posee el $52 \%$ y $50 \%$ de las manzanas con orientación entre $75^{\circ}$ y $105^{\circ}$, seguida por Guaymallén con el 42\%. Las manzanas con mayor porcentaje en la Categoría 6 se ubican en Las Heras $(33.6 \%)$ y Capital $(28.3 \%)$. Estos departamentos poseen las manzanas con las orientaciones extremas (óptimas y desfavorables).

Al sumar la Categoría 5 y 6 el $40.2 \%$ de las manzanas del AMM poseen una orientación entre $0^{\circ}$ y $30^{\circ}$ y entre $150^{\circ}$ y $180^{\circ}$ el mayor porcentaje se ubica en Luján de Cuyo (42.2\%), Godoy Cruz (41.8\%) y Las Heras (41.6\%), mientras el menor porcentaje en Capital (37.9\%) y Guaymallén (38.1\%).

\subsection{RELACIÓN FORMA Y ORIENTACIÓN DE MANZANAS}

Existe asociación entre las variables forma y orientación, p-valor $<0.0001$ (se excluye la Categoría 0 en ambas variables). En la Tabla 11 se detallan los resultados de ambas variables categorizadas.

Tabla 11: Frecuencias absolutas. Fuente: Elaboración propia.

\begin{tabular}{|c|c|c|c|c|c|c|c|c|}
\hline Forma/Orientación & 0 & 1 & 2 & 3 & 4 & 5 & 6 & Total \\
\hline 0 & 9 & 0 & 0 & 0 & 0 & 0 & 0 & 9 \\
\hline 1 & 0 & 1082 & 282 & 73 & 0 & 0 & 0 & 1437 \\
\hline 2 & 0 & 1420 & 449 & 186 & 206 & 751 & 1236 & 4248 \\
\hline 3 & 0 & 786 & 327 & 107 & 77 & 466 & 720 & 2483 \\
\hline 4 & 0 & 436 & 161 & 33 & 34 & 217 & 335 & 1216 \\
\hline+5 & 0 & 334 & 132 & 42 & 37 & 194 & 258 & 997 \\
\hline Total & 9 & 4058 & 1351 & 441 & 354 & 1628 & 2549 & 10390 \\
\hline
\end{tabular}

Con respecto a las manzanas de forma cuadrada el $75.30 \%$ posee una orientación óptima respecto al norte, el $19.62 \%$ orientación Categoría 2 de $105^{\circ}$ a $120^{\circ}$, de $75^{\circ}$ a $60^{\circ}$, de $150^{\circ}$ a $165^{\circ}$ y de $30^{\circ}$ a $15^{\circ}$ y el $5.08 \%$ orientación Categoría 3 de $120^{\circ}$ a $150^{\circ}$ y de $30^{\circ}$ a 60.

Para el resto de las formas de manzanas (excluyendo la Categoría 1) los resultados son los siguientes: el $66.7 \%$ de las manzanas están fuera del valor óptimo de orientación, el 33.3\% poseen una orientación óptima (2976 manzanas). El 28.50\% se orientan en la opción más desfavorable (2549 manzanas).

Para la forma Categoría 5 (la más alargada) el 33.5\% de las manzanas (334 unidades) poseen orientación óptima y para la forma Categoría 4 el 35.9\% de las manzanas (436 unidades), el restante $66.5 \%$ y $64.1 \%$ poseen orientaciones de $0^{\circ}$ a $75^{\circ}$ y de $105^{\circ}$ a $180^{\circ}$ respectivamente.

Las Heras es el departamento que posee la mayor cantidad de sus manzanas de forma 5 con la orientación más desfavorable.

Del análisis departamental de las manzanas con tipología más alargada $>4.25$, puede observarse que poseen una orientación óptima el $24.50 \%$ en Godoy Cruz, el $31.47 \%$ en Maipú 
y el 36.94\% en Luján de Cuyo (el restante $75.50 \%, 68.53 \%, 63.06 \%$ de las manzanas Categoría 5 poseen otras orientaciones).

Si se compara para cada una de las formas 2 a 5, la Categoría orientación 1 con la 6 los valores se aproximan, existiendo mayor cantidad de manzanas en la 1 pero con una diferencia del $7.62 \%$ para la forma $5,8.31 \%$ para la forma $4 ; 2.66 \%$ para la forma 3 y $4.33 \%$ para la forma 2.

La relación forma y orientación de las manzanas urbanas es un elemento regulador de diseño urbano, trabajos previos han determinado una diferencia positiva considerable en la captación solar de manzanas de baja densidad con el eje principal este-oeste, con relación a las manzanas de baja densidad con orientación norte-sur del eje principal, mientras la incidencia de los valores de captación solar en techos no presenta variaciones significativas si se modifica la orientación de la manzana (Figura 18). Para manzanas de media y alta densidad la incidencia de captación solar en muros en relación con techos es aún mayor.

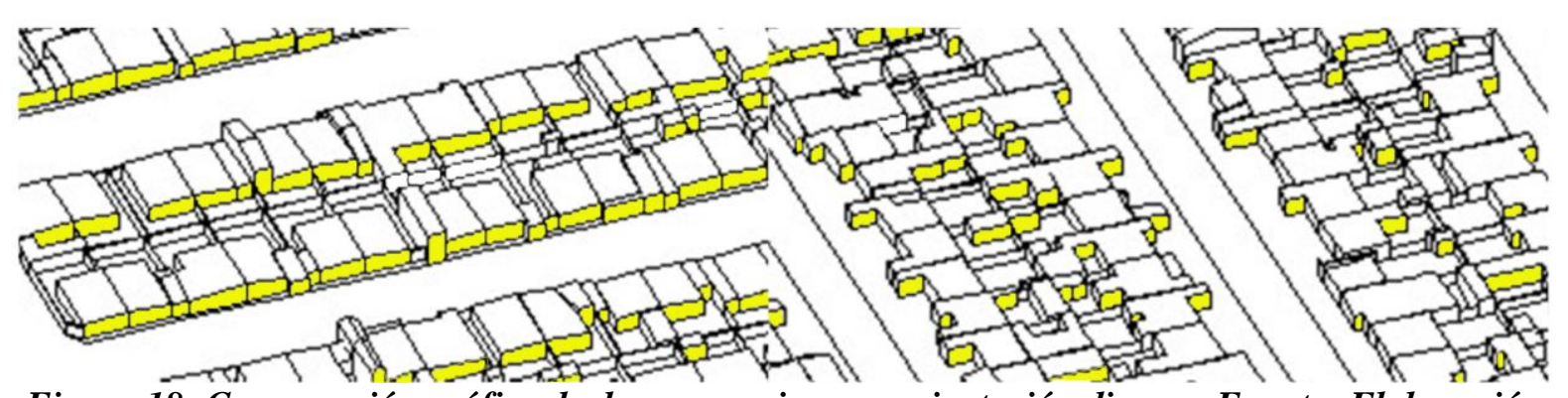

Figura 18: Comparación gráfica de dos escenarios con orientación diversa. Fuente: Elaboración propia.

\section{CONCLUSIONES}

El trabajo profundiza el conocimiento de las variables forma y orientación de las manzanas urbanas que inciden en el potencial solar de entornos urbanos, tomando en consideración la morfología urbana actual y muestra la posibilidad de ser considerados en futuras estrategias de diseño. Ante la posibilidad de aprovechamiento del recurso solar, las configuraciones urbanas presentan limitaciones propias relacionadas principalmente con la forma y orientación de las manzanas, que condicionan la potencialidad colectora solar.

Con los resultados de esta investigación se intenta realizar, desde el ámbito local, un aporte preciso al conocimiento de la profusa y compleja problemática de la sostenibilidad del desarrollo de la sociedad humana con relación a su soporte ecológico, apuntando a producir un impacto positivo, aunque limitado, en la escala global, en términos de ahorro de recursos no-renovables y reducción de emisiones de gases de efecto invernadero.

Enfocando luego sobre aspectos específicos de esa problemática, se verifica que los problemas interrelacionados del hábitat y la energía adquieren una importancia fundamental en dicha escala, ya que se encuentran comprendidos entre los que tienen una gravitación dominante en las condiciones de la sostenibilidad del desarrollo; ellos son: el crecimiento de la población mundial, la creciente tasa de urbanización, el uso intensivo de los recursos naturales no-renovables, la emisión de deshechos contaminantes y la calidad de vida no equitativa de las poblaciones urbanas; todos ellos susceptibles de ser impactados positivamente mediante la implementación de propuestas operativas basadas en resultados, a nivel regional y local.

Considerando ahora las conclusiones específicamente técnicas del estudio realizado, son pertinentes los siguientes comentarios: 
1. Las variables morfológicas de las configuraciones urbanas tienen una incidencia fundamental en la factibilidad técnica de implementar estrategias de aprovechamiento de la energía solar en medios urbanos. Los usos de calefacción solar pasiva y activa de espacios y calentamiento solar de agua sanitaria, son adecuados para aprovechar "in situ" el único recurso energético natural disponible sobre el medio urbano del AMM que presenta, además, otras ventajas apreciables, tales como el llegar a la superficie uniformemente distribuido, ser inagotable y no-contaminante.

2. Las configuraciones urbanas existentes analizadas en el Área Metropolitana de Mendoza $(\mathrm{AMM})$, presentan características diferenciales importantes con respecto a variables urbanas; siendo la morfología de las unidades que constituyen el tejido urbano (manzanas) y la orientación de sus lados de máxima importancia, a los fines de implementar el uso eficiente de la energía solar. Las configuraciones rectangulares en sentido E-O, resultan en el aprovechamiento más eficiente del terreno manteniendo buenas posibilidades de asoleamiento pleno, sobre fachadas al norte (calefacción de espacios) y techos horizontales y en pendiente (calentamiento de agua). Las manzanas de forma Categoría 1: < 1.25, típicas de la trama fundacional, demuestran ser las menos eficientes considerando el acceso al sol, aun estando correctamente orientadas (Arboit, de Rosa, 2013b); pero con una ventaja en cuanto a la conservación energética.

3. La orientación de las manzanas, condiciona las posibilidades de orientación de los edificios que contienen y es un aspecto de máxima importancia para la implementación eficiente de colectores solares para los usos mencionados. Como es sabido, los espacios principales de una vivienda y sus aventanamientos, captarán un máximo de energía radiante cuando están orientados exactamente al norte geográfico. Desviaciones angulares de hasta $15^{\circ}$ al este o al oeste resultarán tolerables, pero más allá de esos valores se sumarán los efectos negativos de: menor intensidad de la energía recibida, durante menos horas al día y con ángulos de incidencia mayores que determinan un incremento de la porción reflejada y una reducción de la absorbida por las superficies vidriadas de los colectores. Es interesante observar aquí que, el costo inicial de un edificio bien orientado es exactamente igual al del mismo edificio mal orientado, pero las diferencias en los consumos de energía durante el ciclo operativo de ambos totalizarán ingentes cantidades de recursos, energía y gases de efecto invernadero. Este principio elemental no ha sido todavía legislado ni implementado como prioritario por los profesionales, ni por los organismos de gestión en la producción del hábitat en la región (Arboit, de Rosa, 2013b).

Las manzanas urbanas cuya proporción entre lados es diferente, presentan gran variación de superficies asoleadas en función de la orientación. Los valores más altos de asoleamiento en fachadas norte corresponden a las tipologías con orientación del lado mayor con dirección EO (Categoría 1), mientras que se presentan reducciones significativas de dichos valores en manzanas Categoría 6.

En la relación entre las variables forma y orientación considerando la forma Categoría 5 (más rectangular) y la orientación Categoría 1 (lado mayor hacia el Norte) el departamento con mayor porcentaje de manzanas que combina forma y orientación óptima es Capital (5.26\%), seguida por Luján de Cuyo (4.30\%), Maipú (3.95\%) y Las Heras (3.80\%). El departamento que deberían priorizar dicha relación es Guaymallén $(3.00 \%)$ además posee la mayor pendiente de expansión urbana (período 1970 -2017), crecimiento población urbana (período 1970 - 2010) y tendencia ascendente de consumo de energía (Molina, Arboit, \& Sedevich, 2019), aunque propiciar dicha combinación sería una estrategia para el aprovechamiento energético solar en todos los departamentos.

Al considerar las formas Categoría 2 a la 5 y con orientación óptima; Godoy Cruz es el departamento que presenta el menor porcentaje de manzanas con dicha orientación (25.78\%), seguida de Luján de Cuyo (29.14\%), Maipú (29.15\%) y Guaymallén (33.17\%), por lo tanto, 
departamentos prioritarios a intervenir por las dificultades que presentan en su trama urbana. Siempre sin considerar las manzanas de forma cuadrada $<1.25$, los departamentos de Las Heras $(44.30 \%)$ y Capital (41.69\% de sus manzanas) poseen la mejor orientación y por tanto donde se podría intervenir prioritariamente con estrategias de calefacción solar pasiva y activa de espacios considerando fachadas norte asoleadas.

Se concluye además que en los aspectos urbanísticos varias estrategias deben ser implementadas para posibilitar la inserción de nuevas construcciones energética y ambientalmente sostenibles, que prioricen el aprovechamiento del recurso solar: entre ellas la forma y orientación de las manzanas son fundamentales para poder ofrecer acceso al sol irrestricto, tanto para calefacción de espacios como para calentamiento de agua, a todas las unidades edilicias.

Con respecto a los aspectos legales del "derecho al sol", existen ya los estudios técnicos y el marco jurídico que justifican la promulgación de una ley que preserve el libre acceso al recurso solar en medios urbanos. Sin embargo, para que esto se materialice es imprescindible contar con la voluntad expresa de los entes gubernamentales involucrados y la reinterpretación de algunas reglamentaciones. La tarea pendiente en este campo es la de incorporar dicha problemática jurídica a los códigos urbanos y de edificación vigentes en los municipios del AMM, en los códigos de toda la Provincia de Mendoza y en las Leyes de Loteo.

El trabajo demuestra que el AMM posee un trazado heterogéneo, los resultados deberían a futuro incorporar otras variables de análisis: económicas, socio-culturales y técnicoambientales; tomando en cuenta los riesgos sísmico y aluvional (terrenos con pendientes abruptas, principalmente pedemontanos, rompen el drenaje hídrico natural que, si no se acompañan con las obras de ingeniería correspondientes, pueden favorecer aluviones); demanda de vivienda, cambios de uso del suelo y nuevas tendencias culturales de la población.

Por otro lado, el estudio espera a futuro poder integrar en un modelo teórico general las variables principales de la morfología urbano-edilicia: forma de las manzana, orientación de las manzanas, ancho de cañón urbano, dispersión de manzanas urbanas, factor de forma (FF) de las manzanas urbanas, plenitud del arbolado, magnitud del arbolado, transmisividad a la radiación solar del arbolado urbano en la estación de invierno y verano, especies arbóreas predominantes, morfologías edilicias, factor de ocupación del suelo (FOS), factor de ocupación total (FOT), factor de forma (FF) de los edificios, altura de edificación (valor promedio por manzana y valores de cada edificio), densidad edilicia, orientación de los edificios, dispersión edilicia (distancia entre edificaciones), relación altura edilicia/ distancia entre edificaciones, entre otras.

Considerando el aspecto metodológico, fue posible elaborar una base de datos y documentos cartográficos en formato GIS de consulta (de aplicación para áreas gubernamentales de planificación urbana-territorial), proporcionando mapas dentro de la ciudad que permitirán (en el avance de la investigación), elaborar correlaciones con otras variables urbano-edilicias, con indicadores energético-ambientales y realizar evaluaciones de servicios ecosistémicos. Las conclusiones, por su parte, lograron establecer relaciones a escala edilicia y urbana.

Se espera, con el avance del estudio, establecer la optimización del aprovechamiento solar para para diferentes morfologías urbano-edilicias y la elaboración de lineamientos de diseño apropiados para la implementación de una legislación que regule el aprovechamiento pleno del recurso solar en la cuidad. 
Los resultados de esta investigación, intentan realizar, desde el ámbito local, un aporte preciso al conocimiento de la profusa y compleja problemática de la sostenibilidad del desarrollo de la sociedad humana con relación a su soporte ecológico, apuntando a producir un impacto positivo, aunque limitado, en la escala global, en términos de ahorro de recursos norenovables y reducción de emisiones de gases de efecto invernadero. Los resultados alcanzados en el trabajo realizado constituyen aportes específicos, limitados al conocimiento de los aspectos técnicos de la problemática de las relaciones entre el hábitat urbano y la energía en la escala local y regional. Si bien la factibilidad técnica de la implementación generalizada del uso de la energía solar en entornos urbanos ya ha sido básicamente demostrada, varios aspectos técnicos deben continuar siendo estudiados en profundidad para consolidar las certidumbres con vistas a posibilitar transferencias masivas. Además, cabe señalar el aporte hacia el logro de una transición energética sostenible, en la que nuestro país está comprometido desde la firma del Acuerdo de París y los Objetivos del Desarrollo Sostenible de la Agenda 2030, a escala global. El muy conocido lema "Pensar globalmente y actuar localmente" provee al trabajo presentado el respaldo conceptual indispensable para que sea considerado como un aporte en la escala local, en el momento actual, ante la evidencia de la magnitud de los problemas que el futuro cercano nos tiene deparados, de no mediar grandes cambios en el corto plazo.

\section{REFERENCIAS BIBLIOGRÁFICAS}

ARBOIT, M., DE ROSA, C. (2013a). Lineamientos y estrategias para la sostenibilidad energética del sector edilicio urbano en zonas de climas secos. Evaluación comparativa de la actualización del Código Urbano y de Edificación de la Ciudad de Mendoza, Argentina. Revista de Urbanismo, 28, 94-113. DOI: 10.5354/07175051.2013.21561

ARBOIT, M., DE ROSA, C. (2013b). Trazado urbano para la sostenibilidad energético ambiental del parquet edilicio en ciudades andinas. Caso: Área Metropolitanade Mendoza, Argentina. Energías Renovables y Medio Ambiente (ERMA), 29, Disponible en: http://erma.asades.org.ar/ojs8/index.php/ERMA/article/view/53>.

ARBOIT, M., DIBLASI, A., FERNÁNDEZ LLANO, J. C., DE ROSA, C. (2008). Assessing the solar potential of low density urban environments in andean cities with desert climates - The case of the city of Mendoza, in Argentina. Renewable Energy, 33 (8), 1733-1748. https://doi.org/10.1016/j.renene.2007.11.007

ARBOIT, M., MESA, A., DIBLASI, A., FERNANDEZ LLANO, J. C., DE ROSA, C. (2010). Assessing the solar potential of low density urban environments in andean cities with desert climates - The case of the city of Mendoza, in Argentina. 2nd. Part. Renewable Energy, 35 (7), 1551-1558. https://doi.org/10.1016/j.renene.2009.11.027

BASSO, M., FERNÁNDEZ, J. C., MESA, N. A., CÓRICA, M. L., DE ROSA, C. (2003). Urban morphology and solar potential of the built environment in Andean Cities of Hispanic Layout. Assessing proposals towards a more sustainable energy future". 20th Conference on Passive and Low Energy Architecture, PLEA 2003, Santiago - Chile.

BREHENY, M. (1996). Centrists, decentrists and compromisers: views on the future of the urban form. En: Jenks, M.; Burton \& E. Williams K. The Compact city: a sustainable urban form? (13-35). London: E \& FN Spon. 
DI RIENZO, J. A., CASANOVES, F., BALZARINI, M. G., GONZALEZ, L., TABLADA, M., ROBLEDO, C. W. (2018). InfoStat versión 2018. Centro de Transferencia InfoStat, FCA, Universidad Nacional de Córdoba, Argentina. URL http://www.infostat.com.ar

DIRECCIÓN GENERAL DE CATASTRO MENDOZA [en línea]. [Consultado 1 junio 2010]. Recuperado de: https://www.atm.mendoza.gov.ar/portalatm/zoneTop/catastro/ catastro.jsp

DROEGE, P. (2007). The Renewable City: A comprehensive guide to an urban revolution, UK: Wiley \& Sons, Incorporated, John.

GIVONI, B. (1998). Climate considerations in building and urban design. USA: John Wiley \& Sons, Inc.

JONES, P., WILLIAMS, J., LANNON, S. (2000). Planning for a sustainable city: An energy and environmental prediction model. Journal of Environmental Planning and Management, 43 (6), 855-872. https://doi.org/10.1080/09640560020001728

MOLINA, G., ARBOIT, M. \& SEDEVICH, A. (2019). Mendoza, ciudad compacta versus metrópolis sin oasis. ISUF-H 2019 III Congreso Internacional Ciudad compacta verus ciudad difusa.

MUTANI, G., FONTANIVE, M., ARBOIT, M. (2018). Energy-use modelling for residential buildings in the metropolitan area of Gran Mendoza (AR). Italian Journal of Engineering Science: Tecnica Italiana., 61 (2), 74-82. Recuperado de: http://iieta.org/sites/default/files/Journals/TI-IJES/61+1.02_04.pdf

OWENS, S. (1986). Energy, planning and urban form. London: Pion Ltd.

R Core Team (2018). R: A language and environment for statistical computing. R Foundation for Statistical Computing, Vienna, Austria. URL https://www.R-project.org/

RATTI C., RAYDAN D., STEEMERS K. (2003). Building form and environmental performance: archetypes, analysis and arid climate, Energy Buildings 35, 49-59. https://doi.org/10.1016/S0378-7788(02)00079-8

STEEMERS, K. (2003). Energy and the city: density, buildings and transport, Energy Buildings, 35, 3-14. https://doi.org/10.1016/S0378-7788(02)00075-0 